\title{
Monitoring von Sprengerschütterungen mit seismischen „low-cost" Sensoren
}

\author{
Ewald Brückl ${ }^{1,2}$, Karl Filz ${ }^{3}$, Roland Hochwartner ${ }^{4}$, Stefan Mertl ${ }^{5}$, Gerald Stickler ${ }^{3}$ und Stefan Zöhling ${ }^{4}$ \\ 'Department für Geodäsie und Geoinformation, Technische Universität Wien, Wien, Österreich \\ ${ }^{2}$ Wien, Österreich \\ ${ }^{3}$ Abteilung Elektrotechnik, Höhere technische Bundes-Lehr- und Versuchsanstalt Wiener Neustadt, Wiener \\ Neustadt, Österreich \\ ${ }^{4}$ Baumit $\mathrm{GmbH}$, Waldegg, Österreich \\ ${ }^{5}$ Mertl Research $\mathrm{GmbH}$, Wien, Österreich
}

Online publiziert 5. September 2019

\begin{abstract}
Zusammenfassung: In den Jahren 2017 und 2018 wurde im südlichen Wiener Becken ein Netz von seismischen „low-cost" Sensoren zur Erfassung lokaler Erdbeben eingerichtet. In einem Umkreis von etwa $10 \mathrm{~km}$ erfassen diese Sensoren auch die von Gewinnungssprengungen im Steinbruch Dürnbach, Hohe Wand ausgehenden seismischen Wellen. Vergleichsmessungen mit einem geeichten Messsystem belegen die Eignung der Daten für das seismische Monitoring.

Der Abfall der seismischen Amplituden mit der Entfernung wird durch ein Potenzgesetz und lokale Verstärkungsfaktoren beschrieben. Letztere variieren nahezu um eine ganze Größenordnung und geben Auskunft, wie repräsentativ die Messwerte an einer einzelnen Station für das gesamte Umfeld sind. Wir quantifizieren die Quellstärke einer Sprengung durch das in der Seismologie übliche Maß der Magnitude. Da wir diese aus den Daten des gesamten Messnetzes ableiten, wird der Einfluss der variablen Abstrahlcharakteristiken der jeweiligen Sprengungen weitgehend eliminiert. Die solcherart berechnete Magnitude bietet sich als Kennwert für die Optimierung der Sprengparameter hinsichtlich einer wirtschaftlich vertretbaren Reduktion der Sprengerschütterungen an. Abschließend zeigen wir, als Entscheidungsgrundlage für die weitere Verdichtung des low-cost Sensor Netzes, in einem Diagramm die Bandbreite der Erschütterungen, mit der bei einer bestimmten Magnitude einer Sprengung im Umfeld gerechnet werden muss.
\end{abstract}

Schlüsselwörter: Sprengerschütterungen, Seismische low-cost Sensoren, Monitoring

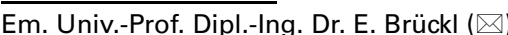

Department für Geodäsie und Geoinformation

Technische Universität Wien,

Wien, Österreich

Ewald.Brueckl@geo.tuwien.ac.at
Monitoring of Blast Vibrations with Seismic Low-Cost Sensors

Abstract: A low-cost seismic sensor network to monitor local earthquakes was installed in the area of the southern Vienna basin in the years 2017 and 2018. These sensors also record seismic waves generated by production blasts in the quarry Dürnbach, Hohe Wand within a radius of about $10 \mathrm{~km}$. Comparative measurements with a calibrated instrument prove the applicability of this data to seismic monitoring.

We describe the decay of the seismic amplitudes with distance according to a power law and local amplification factors. These factors vary within a range of nearly one order of magnitude. They provide information about how representative data from a specific station are for the whole area surrounding the quarry. We quantify the source strength of a quarry blast by the magnitude, a measure commonly used in seismology. Data from all low-cost sensor stations are included in the calculation of the magnitude, thereby eliminating the influence of the varying radiation pattern of the quarry blasts to a large extent. We deem this magnitude an appropriate parameter with respect to the optimization of the blast design to reduce blast vibrations within economically justifiable bounds. Finally, we present a diagram visualizing the magnitude range of ground vibrations one must expect at different distances for a given specific magnitude of the quarry blast. This diagram may serve as a basis for decisions on how and where to build up the existing low-cost sensor network.

Keywords: Blast vibrations, Seismic low-cost sensors, Monitoring 


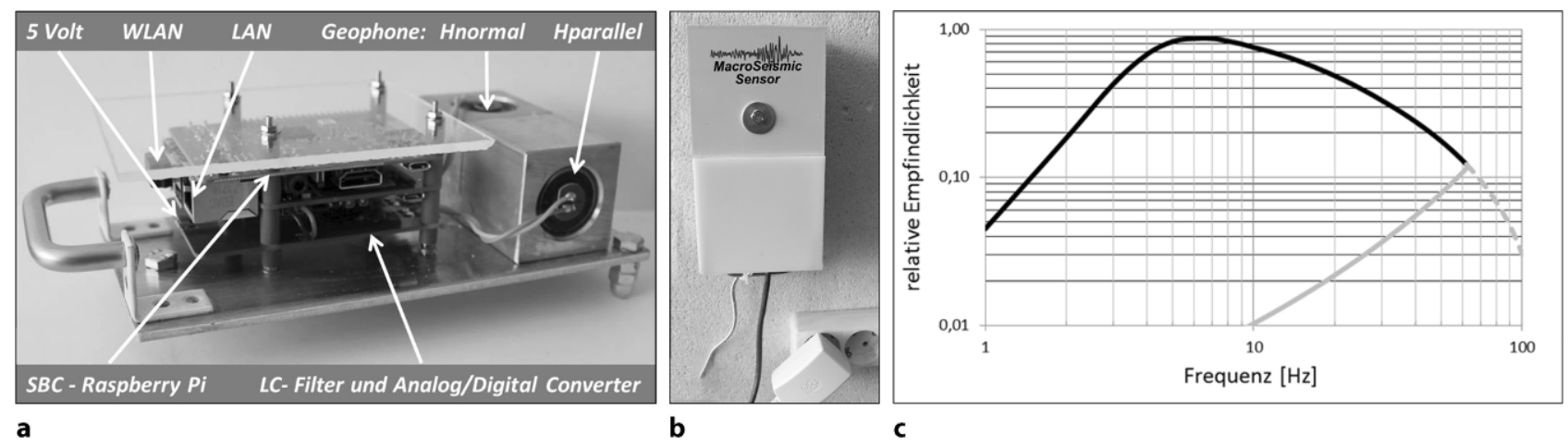

Abb. 1: MacroSeismic Sensor. a Aufbau (ohne Gehäuse). b Montage an einer (tragenden) Wand. c Relative Frequenzcharakteristik (schwarze Linie); Signalanteile über der Nyquist Frequenz (grau strichlierte Linie) erzeugen durch den Alias-Effekt Signalanteile darunter (graue Linie)

\section{Einleitung}

Mit der Einführung von Beschleunigungssensoren auf der Basis von MEMS (Micro-Electro-Mechanical-Systems) in Industrie und privaten Konsum sanken deren Kosten drastisch, sodass sich ihre Verwendung als seismische "lowcost" Sensoren anbot. Als ein Beispiel sei das Projekt „Quake Catcher Network" genannt, im Rahmen dessen ein weltweites Netz aufgebaut werden konnte [1]. Eine qualitativ hochwertige Alternative zu MEMS Sensoren bieten klassische Geophone. Die Initiative "RaspberryShake“ entwickelte mit diesen Sensoren seismische low-cost Messsysteme und konnte diese mittlerweile ebenfalls weltweit verteilen [2]. In Österreich gaben die Sparkling Science und Citizen Science Projekte "Schools \& Quakes" und "QuakeWatch Austria“ Anlass zur Entwicklung eines eigenen, auf Geophonen basierenden seismischen low-cost Sensors. Dieses Messsystem mit dem Namen "MacroSeismic Sensor" soll in erster Linie Daten zu fühlbaren seismischen Ereignissen in nahezu Echtzeit über eine Internetverbindung liefern.

Das kontinuierliche Monitoring von Sprengerschütterungen mit einem den Anforderungen der ÖNORM S 9020 entsprechenden und an geeignetem Ort aufgestellten Messgerät (im Weiteren als "high-end" Gerät bezeichnet) kann als Stand der Technik angesehen werden. Die Empfindlichkeit des MacroSeismic Sensors ist aber auch ausreichend, um im Umfeld von Steinbrüchen Sprengerschütterungen zu erfassen. Am Beispiel des Steinbruchs Dürnbach wird im Folgenden analysiert, welche verbesserten und zusätzlichen Informationen aus der Kombination der Daten des high-end Gerätes mit jenen eines MacroSeismic Sensor Netzes gewonnen werden können.

\section{Der MacroSeismic Sensor}

Im MacroSeismic Sensor wandeln zwei zueinander orthogonal orientierte Geophone (Eigenfrequenz 4,5 Hz) mechanische Schwingungen in elektrische, der Geschwindigkeit proportionale Signale um. Nach einer Tiefpass-Filterung durch ein einfaches RC-Glied (Grenzfrequenz 12,5 Hz) folgt eine 16 Bit Analog-Digitalwandlung mit einer Abtastrate von $\sim 125 \mathrm{~Hz}$. Die Steuerung des AD-Wandlers, die Formatierung und die Übertragung der Daten über LAN oder

\begin{tabular}{|c|c|c|c|}
\hline Gain & $\begin{array}{l}\text { Ausgangsspannung } \\
\text { Geophon [V] }\end{array}$ & $\begin{array}{l}\text { Auflösung } \\
{[\mu \mathrm{m} / \mathrm{s}]}\end{array}$ & $\begin{array}{l}\text { Messbereich } \\
{[\mathrm{mm} / \mathrm{s}]}\end{array}$ \\
\hline 0,5 & \pm 2 & 2,24 & $\pm 73,4$ \\
\hline 1 & \pm 1 & 1,12 & $\pm 36,7$ \\
\hline 2 & $\pm 0,5$ & 0,56 & $\pm 18,4$ \\
\hline 4 & $\pm 0,25$ & 0,28 & $\pm 9,2$ \\
\hline
\end{tabular}

WLAN an einen Server übernimmt ein Ein-Platinen Computer (Raspberry Pi). Die Zeitsynchronisation wird über das Network Time Protocol (NTP) realisiert. Die MacroSeismic Sensoren werden jeweils an einer Wand montiert, wobei ein Horizontalgeophon normal und das andere parallel zur Wand orientiert ist. Abb. 1 zeigt den MacroSeismic Sensor ohne Gehäuse, mit Gehäuse nach der Montage und seine relative Frequenzcharakteristik. In Tab. 1 sind Angaben über die Auflösung und den Messbereich zusammengefasst.

\section{Monitoring der Gewinnungssprengungen im Steinbruch Dürnbach}

Im Steinbruch Dürnbach (Hohe Wand, Niederösterreich) wird Dachsteinkalk der Schneebergdecke gewonnen. Sprengtechnische Parameter der Gewinnungssprengungen 2018 sind in Tab. 2 zusammengefasst.

Das MacroSeismic Sensor Netz im südlichen Wiener Becken umfasst aktuell 25 Stationen und wird derzeit auf mindestens 45 Stationen ausgebaut. Für das Monitoring der Gewinnungssprengungen im Steinbruch Dürnbach wurden bis zu 12 Stationen, die sich im Umkreis von $10 \mathrm{~km}$ um den Steinbruch befinden, herangezogen (Abb. 2). Die Stationen DUBA, WAPE, HOWA und HOPO sind zusätzlich zu den beiden Horizontalgeophonen auch mit einem Vertikalgeophon ausgestattet. Visualisierungen der Daten des MacroSeismic Sensor Netzes sind allen interessierten Bürgern über eine Homepage (https://www. macroseismicsensor.at/) in nahezu Echtzeit zugänglich.

Für seismische Ereignisse, in unserem Fall Gewinnungssprengungen im Steinbruch Dürnbach, berechnen wir die Peak Ground Velocity (PGV) und für 3-Komponenten-Sta- 


\begin{tabular}{|c|c|c|c|c|}
\hline $\begin{array}{l}\text { Etagenhöhe } \\
\text { (von-bis) }\end{array}$ & $\begin{array}{l}\text { Vorgabe } \\
\text { (von-bis) }\end{array}$ & $\begin{array}{l}\text { Volumen } \\
\text { (Max./Median) }\end{array}$ & $\begin{array}{l}\text { Zündstufen } \\
\text { (Max./Median) }\end{array}$ & $\begin{array}{l}\text { Lademenge/Zündstufe } \\
\text { (Max./Median) }\end{array}$ \\
\hline $12,5-25 m$ & $4-5 m$ & $14.950 / 5330 \mathrm{~m}^{3}$ & $56 / 19$ & $140 / 62 \mathrm{~kg}$ \\
\hline
\end{tabular}

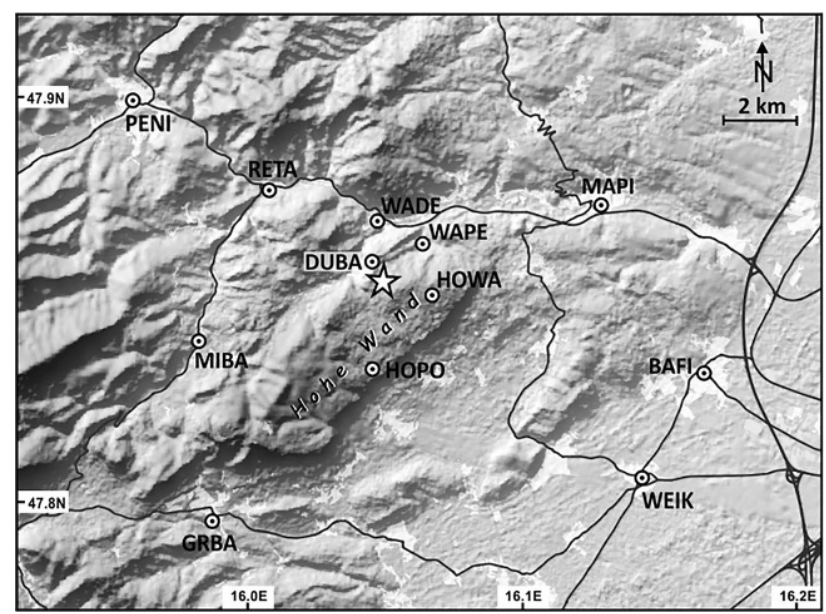

Abb. 2: MacroSeismic Sensor Stationen (BAFI, GRBA ...,WAPE, WEIK) im Umkreis des Steinbruchs Dürnbach (die mittlere Lage der Gewinnungssprengungen ist durch einen Stern markiert), die Karte weist neben der Topographie auch das Straßennetz und besiedelte Flächen aus

tionen auch die resultierende Schwinggeschwindigkeit (VR) nach den GIn. 1 und 2:

$$
\begin{aligned}
& \mathrm{PGV}=\operatorname{Maximum} \sqrt{\left(\mathrm{Hn}(\mathrm{t})^{2}+\mathrm{Hp}(\mathrm{t})^{2}\right)} \\
& \mathrm{VR}=\operatorname{Maximum} \sqrt{\left(\mathrm{Hn}(\mathrm{t})^{2}+\mathrm{Hp}(\mathrm{t})^{2}+\mathrm{Z}(\mathrm{t})^{2}\right)}
\end{aligned}
$$

Das Maximum bezieht sich auf die Zeitdauer des seismischen Ereignisses an den einzelnen Stationen. $\mathrm{Hn}(\mathrm{t})$ und $\mathrm{Hp}(\mathrm{t})$ sind der Zeitverlauf der horizontalen Schwinggeschwindigkeiten normal und parallel zur Wand, an der der Sensor montiert ist. $Z(t)$ entspricht der Vertikalkomponente.

Abb. 3 zeigt die PGV für alle im Jahr 2018 beobachteten Sprengungen über der Schrägdistanz $r$ vom Sprengort zu den verschiedenen Messstellen. Ein im doppelt-logarithmischen Maßstab linearer Zusammenhang von PGV mit $r$ entspricht einem Potenzgesetz (GI. 3):

$$
P G V(r)=A 0 * r^{n}
$$

A0 bedeutet die Quellstärke. Für den Exponenten wählen wir den in der Seismologie gebräuchlichen Wert $n=-1,66$.

\section{Kalibration der MacroSeismic Sensoren}

In der Station DUBA (Keller des Betriebsgebäudes im Steinbruch Dürnbach) sind an einer Fundamentmauer ein MacroSeismic Sensor montiert und am Boden, unmittelbar darunter, das Schwingungsmessgerät Bartec Syscom MR3000TR mit einem externen Geschwindigkeitssensor (Station DUBAM). Dieses Gerät entspricht allen Anforderungen der DIN 45669 und ÖNORM S 9020 und kann als "high-end" Sensor angesehen werden. Abb. 4 zeigt

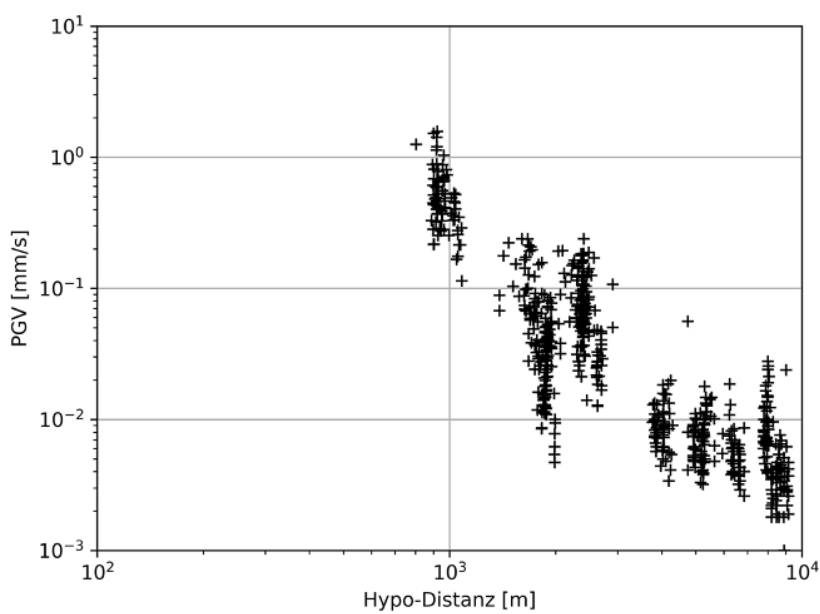

Abb. 3: PGV aller Sprengungen und für jede Station über der Distanz $r$; insgesamt 654 Werte

für die Sprengung vom 15. Oktober 2018 zum Vergleich die von beiden Systemen registrierten Seismogramme, Spektrogramme und die Resultierenden der beiden Horizontalkomponenten.

In Abb. 5 stellen wir das Verhältnis von PGV des highend Sensors zum MacroSeismic Sensor sowohl über der Amplitude als auch dem Datum der Sprengungen dar. Mittelwert und Streuung dieses Verhältnisses betragen: DUBAM_PGV / DUBA_PGV $=1,07 \pm 0,14$. Die Streuung ist mit hoher Wahrscheinlichkeit durch die unterschiedliche Aufstellung, bzw. Ankopplung an das Gebäude (Mauer bzw. Boden) bedingt.

Das Verhältnis von VR zu PGV beträgt für den MacroSeismic Sensor DUBA_VR / DUBA_PGV $=1,07 \pm 0,14$, für den high-end Sensor DUBAM_VR / DUBAM_PGV $=1,03 \pm 0,04$. Wegen der geringen Abweichungen der Daten des MacroSeismic Sensors vom high-end Sensor und der geringen Erhöhung von VR zu PGV betrachten wir im Folgenden nur mehr PGV der MacroSeismic Sensoren.

\section{Lokale Verstärkungsfaktoren und Magnituden}

Die MacroSeismic Sensoren des Messnetzes um den Steinbruch (Abb. 2) wurden in Gebäuden möglichst an tragenden Wänden im Keller, im Parterre oder im ersten Stock montiert. Neben den dadurch gegebenen schwingungstechnischen Unterschieden haben die Bauart der Gebäude, der lokale Untergrund und die geologischen Gegebenheiten Einfluss auf die gemessenen PGV. Da diese Einflüsse von Sprengung zu Sprengung überwiegend gleich bleiben, können sie in Gl. 3 durch stationsspezifische Verstär- 


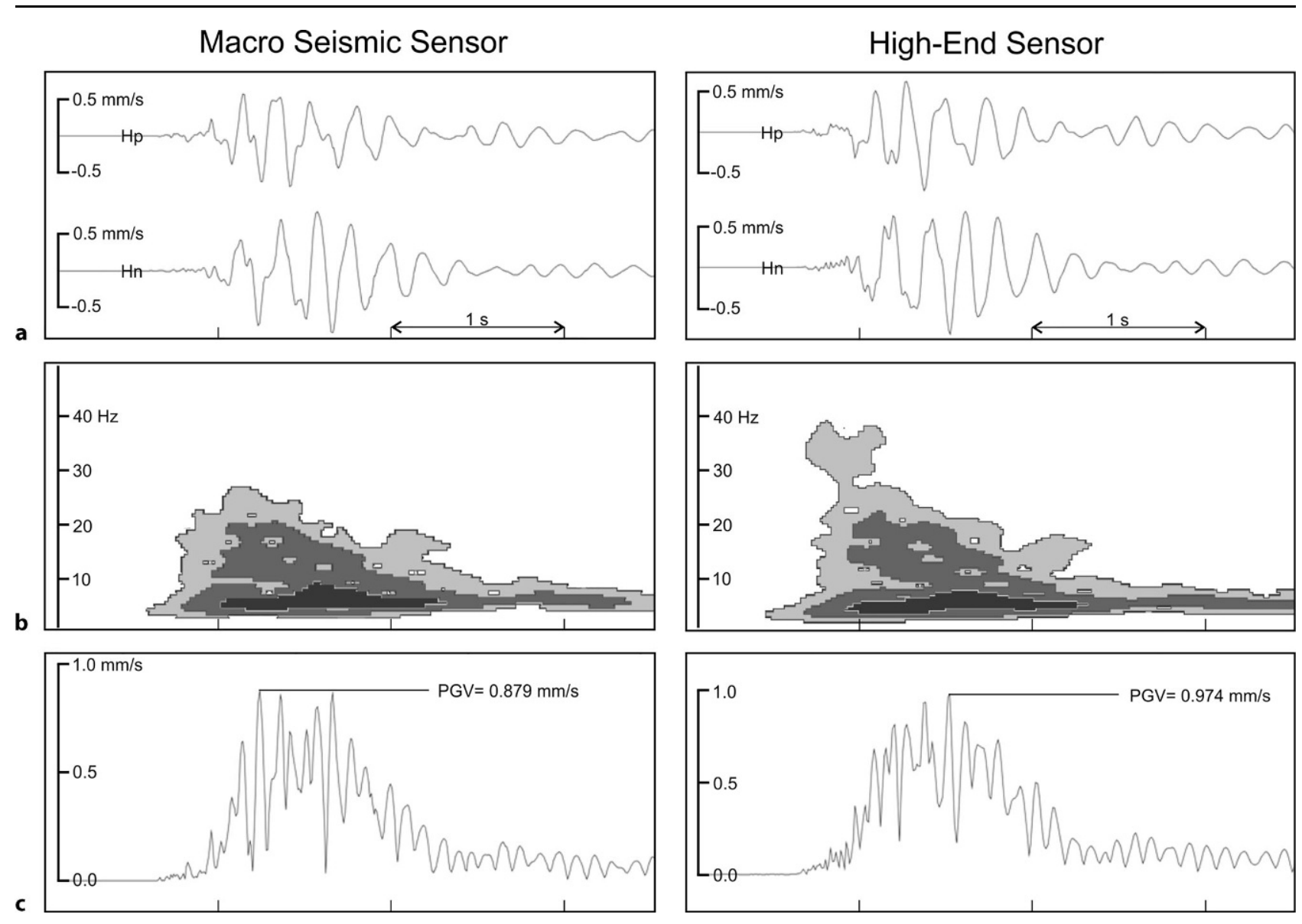

Abb. 4: Sprengung SPR2018073,15. Oktober 2018; Vergleich der Daten des MacroSeismic Sensors mit jenen des high-end Sensors an der Station DUBA. a Seismogramme der Horizontalkomponenten. b Spektrogramme. c Resultierende der Horizontalkomponenten und PGV
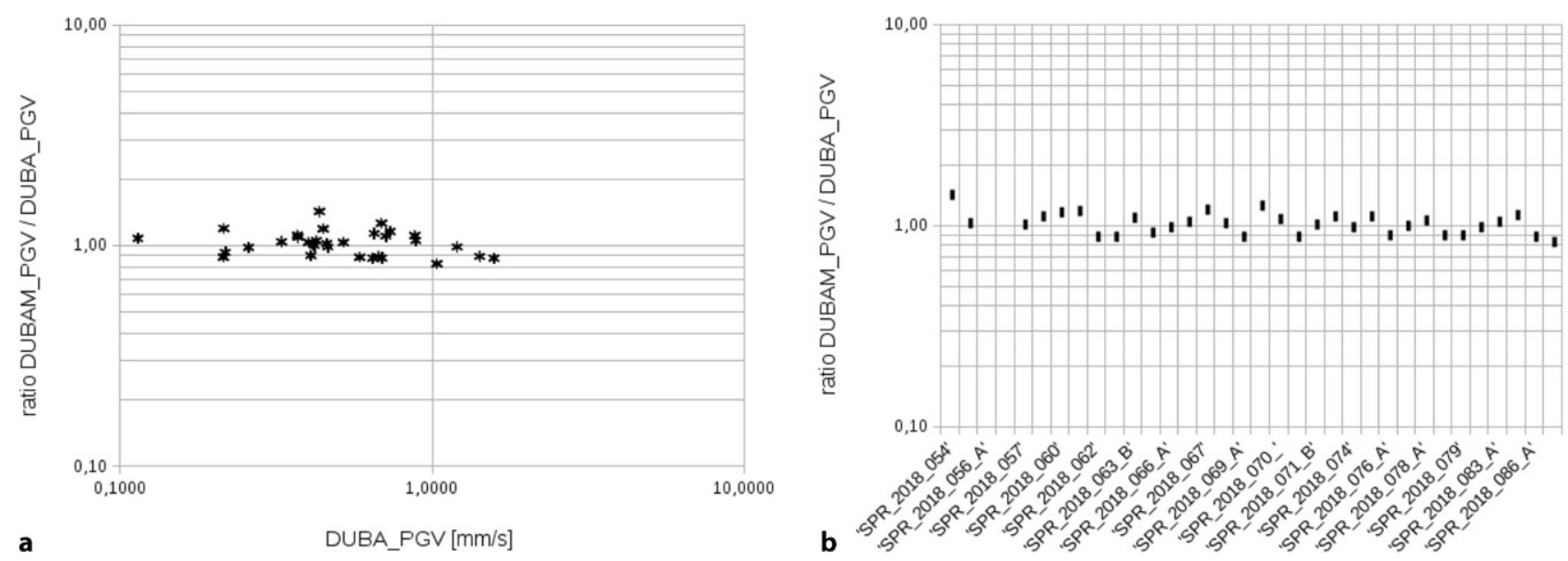

Abb. 5: Verhältnis von PGV (high-end Sensor) zu PGV (MacroSeismic Sensor): a Über der Amplitude. b Über den zeitlich geordneten Sprengungen

kungsfaktoren $\mathrm{SV}_{\mathrm{i}}(\mathrm{i}=1 \ldots N ; N \ldots$ Anzahl der Stationen) berücksichtigt werden (GI. 4):

$$
P G V_{i}=S V_{i} * A 0 * r_{i}^{n}
$$

Wir berechnen für die möglichen Kombinationen der Stationen i und j die geometrischen Mittel von $\mathrm{SV}_{\mathrm{i}} / \mathrm{SV}_{\mathrm{j}}$ über alle bisherigen Beobachtungen mittels der aus der GI. 4 folgenden GI. 5:

$$
\frac{S V_{i}}{S V_{j}}=\frac{P G V_{i} * r_{i}^{-n}}{P G V_{j} * r_{j}^{-n}} \text { füri }=1 \ldots N, j=i+1 \ldots N
$$

Der Logarithmus von GI. 5 ergibt ein lineares Gleichungssystem für SV, das nach der Methode der kleinsten 


\begin{tabular}{|c|c|c|c|c|c|c|c|c|c|c|c|c|}
\hline Station & DUBA & WAPE & HOWA & WADE & HOPO & RETA & MIBA & MAPI & PENI & GRBA & WEIK & BAFI \\
\hline SV & 2,35 & 1,02 & 1,28 & 0,36 & 2,09 & 0,52 & 0,65 & 0,70 & 0,60 & 1,71 & 0,70 & 2,51 \\
\hline Etage & -1 & -1 & -1 & 0 & 0 & 1 & -1 & 0 & 0 & 1 & 1 & 1 \\
\hline $\begin{array}{l}\text { Montage an tragender } \\
\text { Mauer }\end{array}$ & $\mathrm{Ja}$ & $\mathrm{Ja}$ & Nein & $\mathrm{Ja}$ & Nein & $\mathrm{Ja}$ & $\mathrm{Ja}$ & $\mathrm{Ja}$ & $\mathrm{Ja}$ & $\mathrm{Ja}$ & $\mathrm{Ja}$ & $\mathrm{Ja}$ \\
\hline
\end{tabular}

Fehlerquadrate mit der Bedingung, dass das geometrische Mittel aller SV gleich 1 ist, aufgelöst werden kann. Die Ergebnisse sind in Tab. 3, gemeinsam mit zwei schwingungstechnisch relevanten Angaben über die Lage der Sensoren im Gebäude festgehalten.

Ein in der Seismologie übliches Maß für die Stärke einer seismischen Quelle ist die Magnitude. Sie stellt für ein bestimmtes seismisches Ereignis einen Zusammenhang zwischen der an der Station i beobachteten PGV $_{i}$, der Entfernung $r_{i}$ und dem jeweiligen $S V_{i}$ her. Wir berechnen eine spezifische MacroSeismic Sensor Magnitude MSS_Mi mit Gl. 6:

$$
\begin{aligned}
\operatorname{MSSM}_{i}= & \operatorname{LOG} 10\left(\mathrm{PGV}_{\mathrm{i}}\right)-\operatorname{LOG} 10\left(\mathrm{SV}_{\mathrm{i}}\right) \\
& -\mathrm{n}^{*} \operatorname{LOG} 10\left(\mathrm{r}_{\mathrm{i}}\right)+\mathrm{C}
\end{aligned}
$$

Wenn $\mathrm{PGV}_{\mathrm{i}}$ in $\mathrm{nm} / \mathrm{s}$ und die Distanz von der Sprengung zur Station in Grad eingegeben werden, ist $C$ gleich Null. Für den Entfernungsterm gilt weiterhin $n=-1,66$. Der Mittelwert über alle verfügbaren Stationen $\mathrm{i}=1 \ldots N$ ergibt MSS_M. Nach GI. 6 entspricht einer Verdopplung von PGV eine Erhöhung von MSS_M um 0,3.

Abb. 6 zeigt MSS_M der im Jahr 2018 beobachteten Sprengungen. Die Magnituden variieren im Bereich von $1,4 \leq M S S \_M \leq 2,2$. Für die höheren Magnituden sind überwiegend Sprengungen, bei denen auch Sohllöcher verwendet wurden, verantwortlich. Fächersprengungen unterschieden sich nicht signifikant von Sprengungen ohne Sohllöcher.

Die Vergleiche von Abb. 7a mit Abb. 3 und der beiden in Abb. $7 b$ gezeigten Histogramme zeigen, dass durch die Berücksichtigung der Verstärkungsfaktoren SV und die Normierung von PGV auf eine einheitliche Magnitude die Streuung um das in GI. 3 angegebene Potenzgesetz wesentlich reduziert werden konnte. Die Normierung von PGV auf MSS_M $=2,0$ erfolgt durch den aus Gl. 6 folgenden Faktor $10^{\left(2,0-M S S \_M\right)}$.

\section{Schlussfolgerungen}

Entsprechend der Zielsetzung der vorliegenden Studie wolIen wir an Hand der in den Abschn. 4 und 5 dargelegten Ergebnisse analysieren, welche verbesserten und zusätzlichen Informationen aus der Kombination der Daten eines low-cost Sensor Netzes mit jenen eines einzelnen high-end Messsystems gewonnen werden konnten.

Durch Vergleichsmessungen (Abschn. 4) konnte nachgewiesen werden, dass die Daten der MacroSeismic Sensoren in Bezug auf die daraus abgeleiteten Ergebnisse nur vernachlässigbar von den Daten des high-end Gerätes abweichen. Auffallend ist der geringe Unterschied von PGV und VR, also der geringe Einfluss der Z-Komponente des Sensors auf die Resultierende.

Stationsspezifische Verstärkungsfaktoren SV der von den Sprengungen am Ort des Sensors verursachten Erschütterungen beziehen sich auf ein Amplituden - Distanz Gesetz, hier das in GI. 3 angegebene Potenzgesetz mit dem Exponenten $n=-1,66$. Wir präsentieren eine Methode, die die Bestimmung der SV ohne Kenntnis der Quellstärke ermöglicht. Die Verstärkungsfaktoren variieren nahezu innerhalb einer ganzen Größenordnung $(0,36 \leq \mathrm{SV} \leq 2,51)$. Die

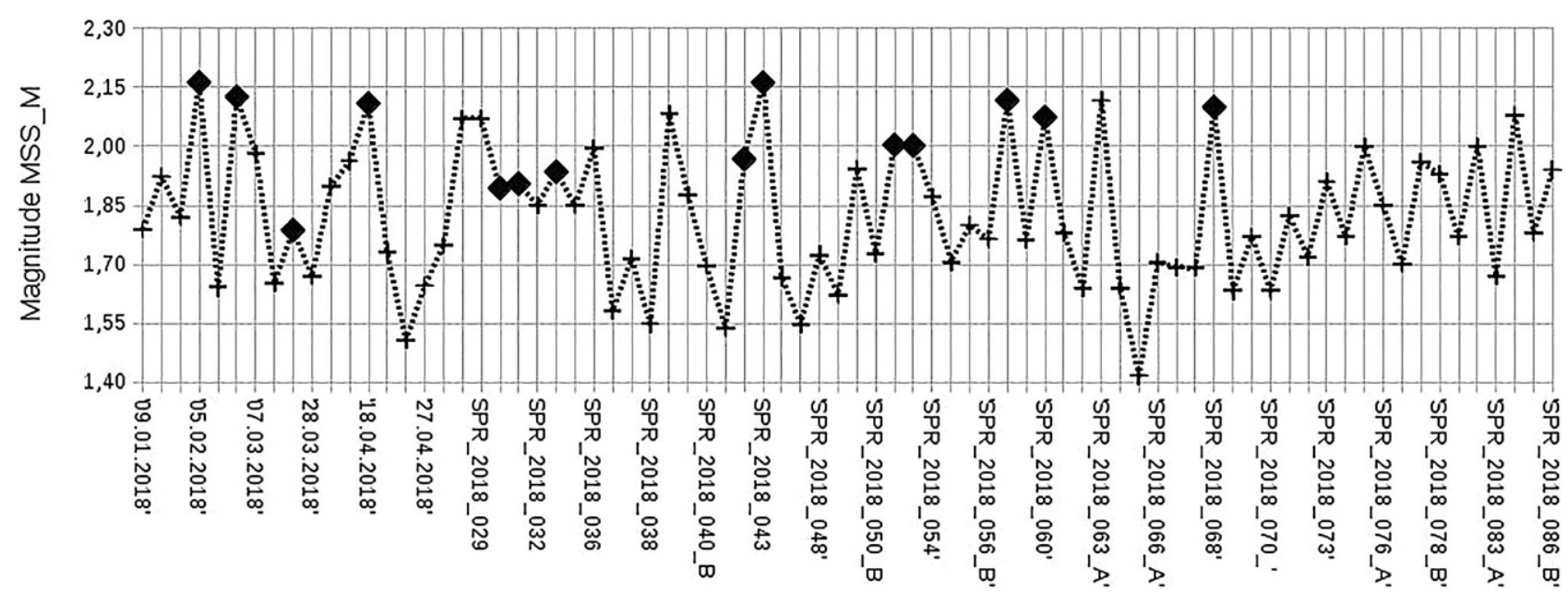

Abb. 6: Magnituden (MSS_M) derim Jahr 2018 beobachteten Gewinnungssprengung (Sprengungen mit Sohllöchern sind durch Rhomben gekennzeichnet) 

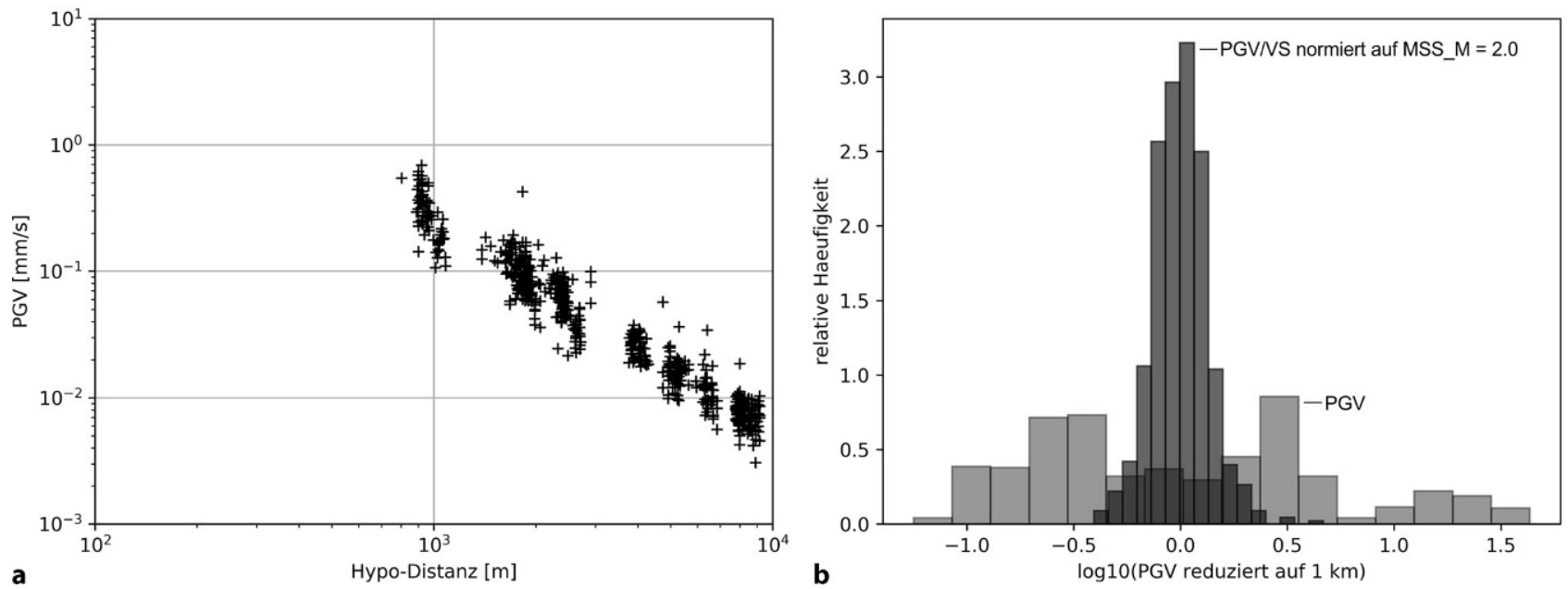

Abb. 7: a PGV mit Berücksichtigung der Verstärkungsfaktoren SV und normiert auf MSS $M=2,0$ über der Distanz. b Histogramme der mit GI. 3 auf die Distanz $r=1 \mathrm{~km}$ reduzierten PGV (Daten in Abb. 3) und PGV / SV normiert auf MSS_M $=2,0$ (Daten in a)

Kenntnis des Verstärkungsfaktors an der Station des highend Sensors und möglichst vielen anderen, die Variabilität der Geologie und Bauweise erfassenden Stationen erlaubt eine Bewertung, wie repräsentativ die Daten des high-end Sensors für das gesamte Umfeld des Steinbruchs sind. Die zeitliche Drift der Empfindlichkeit eines MacroSeismic Sensors bewirkt auch eine Drift von SV an der entsprechenden Station gegenüber der Station mit dem kalibrierten high-end Sensor (hier die Station DUBA). Sie kann dadurch erkannt und korrigiert werden.

Wie in der Seismologie üblich, quantifizieren wir die Quellstärke eines seismischen Ereignisses, in unserem Fall einer Gewinnungssprengung, durch eine Magnitude: der MacroSeismic Sensor Magnitude MSS_M (GI. 6). Die Bestimmung der Magnitude ist umso genauer, je genauer der Abfall der Amplituden mit der Distanz durch das Potenzgesetz (GI. 3) unter der Berücksichtigung lokaler Verstärkungsfaktoren SV beschrieben werden kann. Abb. 7, insbesondere das Histogramm 7b geben einen Aufschluss über die verbleibende Streuung, bzw. den daraus folgenden Fehler in der Bestimmung der Magnitude. Wir nehmen an, dass die verbleibende Streuung überwiegend auf variable Abstrahlcharakteristiken der einzelnen Sprengungen zurückzuführen ist. Bei der Anlage des MSS Netzes um den Steinbruch wurde deshalb versucht, die verschiedenen Abstrahlrichtungen möglichst gut abzudecken, um durch Mittelung über alle stationsspezifischen Magnituden den Einfluss der Abstrahlcharakteristik auf die Bestimmung der Magnitude zu reduzieren.

Abschließend seien in Abb. 8 alle bislang beobachteten PGV normiert auf die Magnitude MSS_M=2,0; jedoch ohne Berücksichtigung der lokalen Verstärkungsfaktoren SV betrachtet. Dieses Diagramm zeigt anschaulich, mit welcher Streuung von PGV in bestimmten Distanzen $r$ auch bei gleicher Quellstärke einer Sprengung zu rechnen ist. Die nach ÖNORM S 9020 als bautechnisch noch irrelevant gewertete $P G V \sim V R=2,5 \mathrm{~mm} / \mathrm{s}$ wird von Sprengungen mit MSS_M $=2,0$ im ungünstigsten Fall bei der Distanz $r=620 \mathrm{~m}$ erreicht. Wegen der großen Variationsbreite der zu prognostizierenden PGV wurde im konkreten Fall beschlossen,

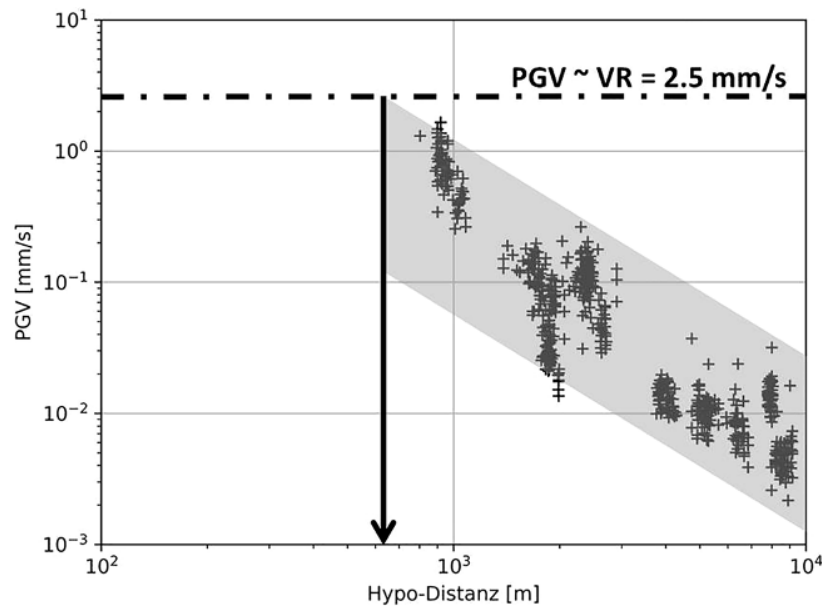

Abb. 8: PGV, normiert auf MSS $M=2,0$ (der mögliche Streubereich von PGV über der Distanz ist durch eine graue Flächegekennzeichnet)

zwei weitere MacroSeismic Sensoren im Nahbereich des Steinbruchs zu installieren.

Danksagung. Besonderen Dank schulden wir den Gemeinden, Schulen und privaten Personen, welche der Installation einer MacroSeismic Sensor Station in ihrem Bereich zustimmten.

Förderung. Der Aufbau des MacroSeismic Sensor Netzes begann mit den Sparkling Science und Citizen Science Projekten „Schools \& Quakes" und „QuakeWatch Austria“, gefördert durch das damalige BMWFW. Der weitere Ausbau wurde und wird von der Niederösterreichischen Landesregierung im Rahmen der Projekte "Aufbau eines Netzes seismischer low-cost Sensoren zur Klassifizierung und Quantifizierung fühlbarer Boden- und Gebäudeerschütterungen im Raum des südlichen Wiener Beckens" und "Seismische Sensoren informieren die Gesellschaft" gefördert.

Funding. Open access funding provided by TU Wien (TUW).

Open Access Dieser Artikel wird unter der Creative Commons Namens nennung 4.0 International Lizenz (http://creativecommons.org/licenses/ by/4.0/deed.de) veröffentlicht, welche die Nutzung, Vervielfältigung, Bearbeitung, Verbreitung und Wiedergabe in jeglichem Medium und Format erlaubt, sofern Sie den/die ursprünglichen Autor(en) und die Quelle 
ordnungsgemäß nennen, einen Link zur Creative Commons Lizenz beifügen und angeben, ob Änderungen vorgenommen wurden.

\section{Literatur}

1. Cochran, E.S.; Lawrence, J.F.; Christensen, C.; Jakka, R.S.: The Quake-Catcher Network: Citizen Science Expanding Seismic Horizons, Seismological Research Letters 80 (2009), no. 1, pp 26-30 (https:// doi.org/10.1785/gssrl.80.1.26)

2. Anthony, R.E.; Ringler, A.T.; Wilson, D.C.; Wolin, W.: Do low-cost seismographs perform well enough for your network? An overview of laboratory tests and field observations of the OSOP Raspberry Shake 4D, Seismological Research Letters 90 (2019), no. 1, pp 219-228 (https://doi.org/10.1785/0220180251)

Hinweis des Verlags. Der Verlag bleibt in Hinblick auf geografische Zuordnungen und Gebietsbezeichnungen in veröffentlichten Karten und Institutsadressen neutral. 